\title{
Modelling the initiation of coronal mass ejections: magnetic flux emergence versus shearing motions
}

\author{
F. P. Zuccarello ${ }^{1,2}$, C. Jacobs ${ }^{1,2}$, A. Soenen ${ }^{1,2}$, S. Poedts ${ }^{1,2}$, B. van der Holst ${ }^{3}$, and F. Zuccarello ${ }^{4}$ \\ ${ }^{1}$ Centre for Plasma-Astrophysics, K. U. Leuven, Celestijnenlaan 200B, 3001 Leuven, Belgium \\ e-mail: Francesco.Zuccarello@wis. kuleuven . be \\ 2 Leuven Mathematical Modelling \& Computational Science Research Centre (LMCC), Belgium \\ 3 Center for Space Environment Modeling, University of Michigan, 2455 Hayward Street, Ann Arbor, MI 48109, USA \\ 4 Dipartimento di Fisica e Astronomia - Universitá di Catania via S.Sofia 78, 95123 Catania, Italy \\ Received 20 May 2009 / Accepted 14 July 2009
}

\section{ABSTRACT}

\begin{abstract}
Context. Coronal mass ejections (CMEs) are enormous expulsions of magnetic flux and plasma from the solar corona into the interplanetary space. These phenomena release a huge amount of energy. It is generally accepted that both photospheric motions and the emergence of new magnetic flux from below the photosphere can put stress on the system and eventually cause a loss of equilibrium resulting in an eruption.

Aims. By means of numerical simulations we investigate both emergence of magnetic flux and shearing motions along the magnetic inversion line as possible driver mechanisms for CMEs. The pre-eruptive region consists of three arcades with alternating magnetic flux polarity, favouring the breakout mechanism.

Methods. The equations of ideal magnetohydrodynamics (MHD) were advanced in time by using a finite volume approach and solved in spherical geometry. The simulation domain covers a meridional plane and reaches from the lower solar corona up to $30 R_{\odot}$. When we applied time-dependent boundary conditions at the inner boundary, the central arcade of the multiflux system expands, leading to the eventual eruption of the top of the helmet streamer. We compare the topological and dynamical evolution of the system when driven by the different boundary conditions. The available free magnetic energy and the possible role of magnetic helicity in the onset of the CME are investigated.

Results. In our simulation setup, both driving mechanisms result in a slow CME. Independent of the driving mechanism, the overall evolution of the system is the same: the actual CME is the detatched helmet streamer. However, the evolution of the central arcade is different in the two cases. The central arcade eventually becomes a flux rope in the shearing case, whereas in the flux emergence case there is no formation of a flux rope. Furthermore, we conclude that magnetic helicity is not crucial to a solar eruption.
\end{abstract}

Key words. Sun: coronal mass ejections (CMEs) - magnetohydrodynamics (MHD) - methods: numerical

\section{Introduction}

Solar phenomena, such as flares and coronal mass ejections (CMEs), are often associated with sheared magnetic fields and the emergence of new magnetic flux. Which of the two is more critical is not yet clear (Liu \& Hayashi 2006). Moreover, the shear may already be present in the active region fields or can be induced by photospheric motions. Observational evidence also suggests that shearing motions along the neutral line seem to play an important role in eruptive events. Large solar flares are typically found to occur along the most highly sheared portions of magnetic inversion lines. Yang et al. (2004) report shear flows prior to an X10 flare, while Falconer (2001) show that CME productivity is strongly correlated with magnetic shear.

On the other hand, Martin et al. (1982) studied the positions of 88 flares and concluded that at least two-thirds of these flares were strictly related to the emergence of new magnetic flux. Green et al. (2003) studied four young active regions and find that the majority of CMEs and flares occurred in those regions during or after new flux emergence. Zhang et al. (2008) published a statistical study on $189 \mathrm{CME}$-source regions, 46 active regions, and 15 newly emerging active regions. They find that $60 \%$ of the CME-source regions show magnetic flux increase during $12 \mathrm{~h}$ before the eruption and $40 \%$ show magnetic flux decrease. Sterling et al. (2007) show that emergence of new magnetic flux is intimately related to the rise of the filament and the eruption of the CME. Observations also show that filament eruptions can account for $75 \%$ of the observed CMEs, in which a clear bipolar magnetic field associated to the filament is observed. However, the origin and the magnetic topology of the other $25 \%$ are not clear yet.

From a theoretical point of view, understanding the origin of CMEs is an open challenge as well. Several papers reviewing the different CME initiation theories and models are available in the literature (e.g. Klimchuk 2001; Zhang \& Low 2005; Forbes et al. 2006; Roussev \& Sokolov 2006). One aspect seems to have gained consensus: whichever CME model one uses, it has to include (1) a way to build up free magnetic energy and (2) some mechanism capable of converting magnetic energy into kinetic energy. According to Klimchuk (2001), the CME initiation models can be characterised in directly driven and storagerelease models. In the first class of models, the magnetic energy pumped into the system is continuously converted into energy of the eruption. In the second class of models, the magnetic energy is first stored and then released. Magnetic energy can be added to the coronal system by means of photospheric motions, shearing, and/or twisting of the magnetic field. The emergence of new magnetic flux can also pump magnetic energy into the system, while flux cancellation processes lead, via reconnection, to a sudden release of this energy. 
The role of photospheric motions has been investigated numerically, for example, by van Ballegooijen \& Martens (1989) and Amari et al. (2003). In these works, the initially potential magnetic field is first subjected to twisting motions, until a more complex structure is reached. Afterwards, converging motions towards the polarity inversion line lead to the formation and destabilisation of the flux rope.

To investigate the dynamics of the coronal mass ejections on 2002 April 21 and August 24, Roussev et al. (2007) performed a numerical simulation in which a reconstructed magnetic field is made unstable by stretching the opposite polarity feet of a newly emerged magnetic dipole. As a result, magnetic flux and helicity are transferred from the expanding flux system, containing the evolving dipole, to the nearby flux systems. Photospheric shearing motions have generally been used as a trigger mechanism in the breakout scenario (Antiochos et al. 1999). This CME initiation model exploits the vulnerability of multipolar topologies to rearrangements of the magnetic field's connectivity, enabling the eruption. The reconnection site is located above the central arcade that reconnects with the overlying field to create a passage for the CME. Subsequently, MacNeice et al. (2005) used more sophisticated numerical techniques to investigate the behaviour of CMEs in a breakout scenario and show that fast CMEs can be produced.

A three-dimensional (3D) simulation of the breakout scenario has recently been performed by Lynch et al. (2008) and DeVore \& Antiochos (2008). The role of flux cancellation mechanisms in a complex multiflux configuration, similar to that of the breakout model, was studied by Amari et al. (2007). Their results show similar features to those in earlier simulations for a bipolar configuration (Amari et al. 2000). The presence of an $\mathrm{X}$-point above the twisted flux rope makes the expulsion of the latter much easier thanks to the weaker confinement near this point.

Archontis et al. (2007) demonstrate that during a multiple flux emergence event from just below the photosphere into a non-magnetised lower corona, a configuration typical of the breakout model can be reached as a result of a diffusive relaxation phase. Rising tubes have also been studied by Fan \& Gibson (2004) showing that a sufficiently twisted flux tube emerging in the solar atmosphere can become kink-unstable and result in an eruption. Recently, Archontis \& Török (2008) simulated the rise of a subphotospheric twisted flux tube into the solar corona. The authors found that a flux rope is formed within the expanding field because of shearing and reconnection of field lines at low atmospheric heights. Depending on whether the corona is magnetised or not, the expanding flux rope experiences a full eruption or remains confined.

An alternative mechanism is the flux injection, or flux emergence model as described by Chen \& Shibata (2000). In this model a pre-existing flux rope, in cartesian geometry, is made unstable by the emergence of new magnetic flux of opposite polarity in the pre-existing coronal loops. This causes a decrease in magnetic pressure and leads to the formation of a current sheet. Dubey et al. (2006) extended this model including geometrical effects and the gravitational stratification of the surrounding medium. In a two dimensional spherical geometry, Ding \& Hu (2008) considered the problem of the equilibrium of a flux rope, embedded in a background wind. Their simulations show that the emergence of new magnetic flux in the background field and far away from the flux rope itself eventually results in its destabilisation. The catastrophic behaviour of the system depends on the location and field orientation of the emerging arcade and on whether magnetic reconnection across the newly formed current sheet takes place.

The aim of this research is a numerical study of the effectiveness of different scenarios for CME initiation in the framework of the breakout model. We focus on the shearing of the magnetic field lines and emergence of new magnetic flux as mechanisms to energise the solar corona. The work presented here builds on the results presented by van der Holst et al. (2007) and Zuccarello et al. (2008). In a previous paper, we showed that the emergence of new magnetic flux of the same sign as that of the central arcade of a breakout configuration can cause CMEs. Van der Holst et al. (2007) studied the role of foot point shearing in the context of the breakout model superimposed on a solar wind solution. Both studies show that the nature of the CME obtained is significantly affected by the presence of the background wind. We present the results of several simulations in which foot point shearing and/or magnetic flux emergence are used as driving mechanisms. The simulations are performed in the physical framework of ideal magnetohydrodynamics (MHD). A steady state solution for the background wind as in van der Holst et al. (2007) and Zuccarello et al. (2008) is reconstructed in a spherical, axial symmetric domain. We then objectively investigate the topological and dynamical evolution of the system when it is subjected to the two driving mechanisms. Since the only difference between the simulations is the CME initiation mechanism, this allows a direct comparison of similarities and differences, possibly observable, when the same system is subjected to different drivers.

The structure of the paper is as follows. A detailed description of the model, the numerical method, and the boundary conditions are given in the next section. In Sect. 3, the morphological and dynamical evolution of the system when it is driven by foot point shearing and/or flux emergence is presented. In Sect. 4 we discuss the results, while conclusions are drawn in Sect. 5.

\section{Description of the numerical model}

The ideal MHD equations are solved in spherical, axisymmetric (2.5D) geometry. The simulation domain covers the region from the lower corona up to $30 R_{\odot}$ and from the solar north to the solar south pole, i.e. $(r, \theta) \in[1,30] \times[0, \pi]$. The computational mesh contains $484 \times 205$ cells for $(r, \theta)$, respectively, including two ghost cells at each end. The grid points are non-uniformly distributed with a finer mesh size around the equator and toward the solar surface. The last-to-first grid cell ratio is 135 for the radial direction, and this ratio is 0.3 for the equatorial to polar cell size. The radial resolution on the solar surface is $1.5 \mathrm{Mm}$, while the angular resolution at the equator is about $0.45^{\circ}$. All the simulations discussed in this paper were performed on exactly the same grid, with the same numerical technique and using the same initial conditions. A modified version of the versatile advection code (VAC) (Tóth 1996) is employed to advance the ideal MHD equations in time. The robust total variation diminishing (TVD) Lax-Friedrichs scheme is used with diffusive but stable minmod slope limiter for second-order reconstructions. Instead of storing the magnetic field components $B_{r}$ and $B_{\theta}$ on a staggered mesh, we use the vector potential component $A_{\phi}$ in the nodes. This approach guarantees a divergence-free solution in the ghost cells as well.

\subsection{Solar wind model}

The ideal MHD equations were solved in a frame corotating with the Sun, resulting in the inclusion of the centrifugal and Coriolis 
Table 1. Wind model parameters.

\begin{tabular}{ccc}
\hline \hline Parameter & $|\pi / 2-\theta|<\theta_{0}$ & $|\pi / 2-\theta|>\theta_{0}$ \\
\hline$q_{0}$ & $10^{6} \mathrm{erg} \mathrm{g}^{-1} \mathrm{~s}^{-1} \mathrm{~K}^{-1}$ & $10^{6} \mathrm{erg} \mathrm{g}^{-1} \mathrm{~s}^{-1} \mathrm{~K}^{-1}$ \\
$T_{0}$ & $1.5 \times 10^{6} \mathrm{~K}$ & $2.63 \times 10^{6} \mathrm{~K}$ \\
$\sigma_{0}$ & $4.5 R_{\odot}$ & $4.5\left[2-\sin ^{2}(\theta) / \sin ^{2}\left(\theta_{0}\right)\right] R_{\odot}$ \\
$\gamma$ & $5 / 3$ & $5 / 3$ \\
\hline
\end{tabular}

forces. The other source terms in our model are the gravitational force and an additional heating/cooling term that mimics the effect of heat conduction and dissipation above the transition region to reproduce a realistic solar wind. For the latter, we used a functional form closely resembling that of Groth et al. (2000) and later described in more detail by Manchester et al. (2004), namely

$Q=\rho q_{0}\left(T_{0}-T\right) \exp \left[\frac{-(r-1)^{2}}{\sigma_{0}^{2}}\right]$,

where $\rho$ is the density, $T$ the temperature, $q_{0}$ the volumetric heating amplitude, $T_{0}$ the target temperature, and $\sigma_{0}$ the heating scale height. The values of these parameters as a function of a critical angle are displayed in Table 1 . For $r<7$, this critical angle is defined by $\sin ^{2} \theta_{0}=\sin ^{2}\left(17.5^{\circ}\right)+\cos ^{2}\left(17.5^{\circ}\right)(r-1) / 8$. For $7<r<30$, this becomes $\sin ^{2} \theta_{0}=\sin ^{2}\left(61.5^{\circ}\right)+\cos ^{2}\left(61.5^{\circ}\right)(r-$ $7) / 40$. Including an artificial heating/cooling term can, and most likely will, quantitatively change the dynamics of the coronal plasma in comparison with the real plasma evolution. The shape and field line topology of the helmet streamer in particular depends on the heating/cooling term. As discussed in van der Holst et al. (2007), the reconnection sites depend on the heating mechanism, but the results do not qualitatively change by changing the heating formulation.

The initial condition corresponds to the 1D hydrodynamic solar wind solution of Parker (1958), combined with a dipole field that has a strength of $2.2 \mathrm{G}$ at the poles. An extra term is added to the vector potential dipole field to obtain the multiflux distribution, suitable for the breakout model to work. The additional term takes the form

$A_{\phi_{\mathrm{ex}}}=A_{0} \frac{\cos ^{2}\left(\frac{\pi \lambda}{2 \Lambda}\right)}{r^{4} \sin \theta}$,

where $\lambda=\pi / 2-\theta$ is the solar latitude, $A_{0}=-0.72 \mathrm{G} R_{\odot}^{5}$, and $\Lambda=0.5$. This field is only added to the dipole field when $|\lambda|<\Lambda$. At the solar base, the density is fixed to $\rho=1.67 \times 10^{-16} \mathrm{~g} \mathrm{~cm}^{-3}$, the temperature is $T=1.5 \times 10^{6} \mathrm{~K}$, the $A_{\phi}$ component is set to the previously stated total vector potential, and the boundary angular velocity is set to zero in the corotating frame. All other values at the solar base, and the outer boundary located at $30 R_{\odot}$, are extrapolated. We let the system evolve to a steady state solution using these boundary conditions. The obtained solar wind consists of the typical two types of solar wind. A region of slow wind is located around the equator with radial outflow between 350 and $400 \mathrm{~km} \mathrm{~s}^{-1}$. The fast wind originates from the polar regions and has a radial outflow of about $710 \mathrm{~km} \mathrm{~s}^{-1}$ (Fig. 1a). The three arcades, which are formed inside the helmet streamer due to the addition of the extra flux in the equatorial region, are shown in Fig. 1b. The field orientation of the central arcade is opposite to that of the overlying streamer and extends approximately 0.155 radians from the equator north- and southward.

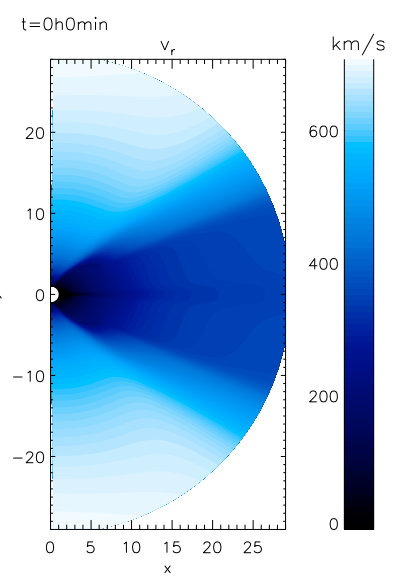

(a)

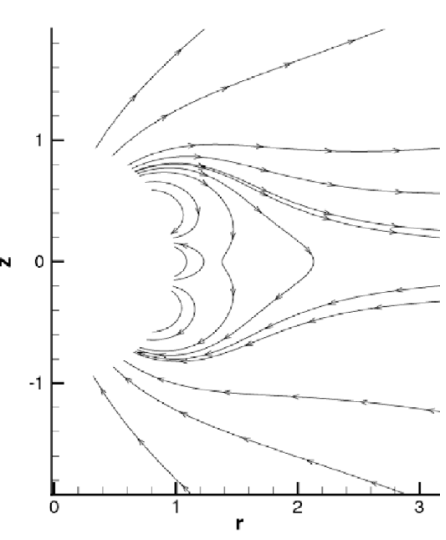

(b)
Fig. 1. Initial steady state configuration. a) The radial component of the velocity field is plotted in colour scale. b) Magnetic field lines showing the helmet streamer and the triple arcade structure suitable for the breakout model.

\subsection{Driving mechanisms}

\subsubsection{Foot point shearing}

As a first driving mechanism of the system we impose a localised shear velocity at the solar base. The shear flow follows the profile:

$v_{\phi}=v_{0}\left(\lambda^{2}-\Delta \theta^{2}\right)^{2} \sin \lambda \sin \left[\pi\left(t-t_{0}\right) / \Delta t\right]$,

when the latitude $\lambda=\pi / 2-\theta$ is in the range $|\lambda|<\Delta \theta$, and $v_{\phi}=0$ otherwise. The shear flow start time is $t_{0}=0$, and the total shear time, $\Delta t$, is approximately $24 \mathrm{~h}$. After time $\Delta t$, we switch off the shear velocity, leaving the central arcade in a sheared state. The shear region is fixed to $\Delta \theta=0.15$ radians, so that we apply our shear profile just inside the central arcade. The maximum shear flow is defined by the value of the amplitude, $v_{0}$, and is $7.6 \mathrm{~km} \mathrm{~s}^{-1}$. We want to stress that this is the value of the shear velocity at time $\Delta t / 2$ and at a latitude of $\pm 4^{\circ}$, so the average shear velocity is much less than this value. This value is still higher than what is observed at the photosphere. However, the inner boundary of our simulation corresponds to the lower corona. This choice for the shearing velocity is a compromise between observational constraints and computational requirements. The maximum shear profile as function of the latitude is plotted in Fig. 2. The effect of the shear flow is an increase in the azimuthal component of the magnetic field. The shearing motions are applied around the solar equator, such that they are localised around the polarity inversion line of the central arcade. The boundary conditions for all the other variables are left unchanged.

\subsubsection{Flux emergence}

The second driving mechanism, flux emergence, is simulated by applying a time-dependent boundary condition on the azimuthal component of the vector potential, taking the form:

$\left.A_{\varphi}(t, \theta)\right|_{r=1}=\left.A_{\varphi}(t=0, \theta)\right|_{r=1}+c_{\mathrm{e}} \frac{\left(t-t_{0}\right)}{\Delta t} \cos ^{2}\left(\frac{\pi \lambda}{2 \Delta \theta}\right) \frac{1}{\sin \theta}$

where $\Delta \theta$ delimits the flux emergence region, $\Delta t=t_{\mathrm{e}}-t_{0}=24 \mathrm{~h}$ is the time interval of flux emergence, and $c_{\mathrm{e}}=-0.9$ is chosen in 


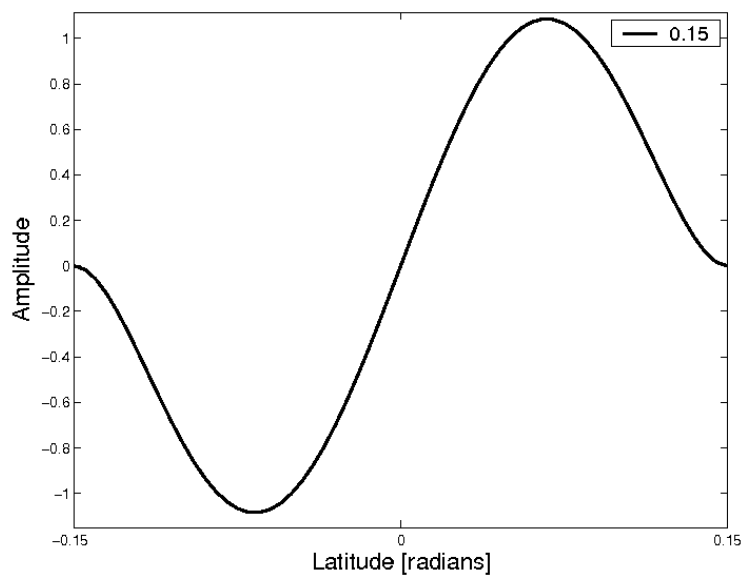

Fig. 2. Shear velocity as a function of the latitude $\lambda=\pi / 2-\theta$. The shear profile is shown at its maximum, viz. when $t=\Delta t / 2$.

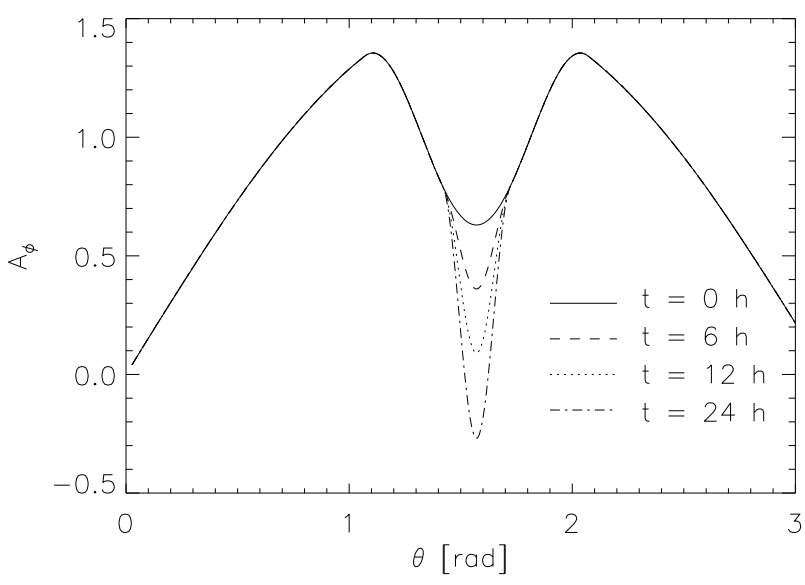

Fig. 3. Azimuthal component of the vector potential at the solar surface $(r=1)$ as a function of $\theta$ at different instants in time. Solid line: the initial state $(t=0)$; dotted line: $t=6 \mathrm{~h}$; dashed line: $t=12 \mathrm{~h}$; dotteddashed line: $t=24 \mathrm{~h}$, this is when all the flux has emerged (Zuccarello et al. 2008).

such a way that, after the time $\Delta t$, the flux of the active region is doubled. All the other boundary conditions remain unchanged; i.e., in this case we keep the azimuthal component of the velocity fixed to zero. Figure 3 shows the azimuthal component of the vector potential at the solar surface as a function of the colatitude $\theta$ and of the time. The solid line represents the initial steady-state magnetic field configuration. The multiflux distribution is evident. The two maxima correspond to the polarity inversion line of the side arcades, while the minimum corresponds to the magnetic neutral line of the central arcade, whose field goes in the opposite direction of both the lateral arcades and the overlying field. The new emerging flux has the same polarity as the pre-existing central arcade flux. The total amount of emerged flux is $\Phi_{\mathrm{E}}=2 \pi\left|c_{\mathrm{e}}\right|=1.97 \times 10^{22} \mathrm{Mx}$, while the flux emergence rate is $4.58 \times 10^{18} \mathrm{Mx} \mathrm{s}^{-1}$. The applied boundary condition on the vector potential only modifies the radial magnetic field, $B_{r}$, at the inner boundary. After time $t_{\mathrm{e}}=24 \mathrm{~h}$, no more flux emerges and the azimuthal component of the vector potential is kept constant at the inner boundary to the value $\left.A_{\varphi}\left(t_{\mathrm{e}}, \theta\right)\right|_{r=1}$.

After time $\Delta t$, in both cases, the system is left to evolve until about $80-90 \mathrm{~h}$ of physical time. We would like to stress that the angular extend over which the shearing motions or the flux emergence are applied is the same in both simulations, viz. $2 \Delta \theta=0.3$ radians, and is embedded completely inside the inner arcade.

\section{Results}

\subsection{Topological evolution}

After the steady state is reached, we impose the time-dependent boundary conditions discussed in the previous section. The evolution of the relative density for shearing and flux emergence cases are shown in Figs. 4 and 5, respectively. Although the initial configuration and the time interval over which the time dependent boundary conditions are applied are identical, the time scales over which the system evolves differ. For this reason, we do not compare the two driving mechanisms at equal time steps, but at the moments where the morphological structure of the system is similar.

Figure $4 \mathrm{a}$ shows a snapshot of the system at time $17 \mathrm{~h} 12 \mathrm{~min}$ where, as a consequence of the shearing motions, the balance between magnetic pressure and magnetic tension is broken. As a result, the central arcade starts to rise up. Figure 5a shows the early stages of the evolution when the system is driven by the emergence of new magnetic flux. In this case, the emerging radial component of the field (Zuccarello et al. 2008) will cause the increase in the magnetic pressure, so that the central arcade will expand in a similar way, but only after about $21 \mathrm{~h}$. Although we apply both shearing and flux emergence over the same region inside the central arcade, the shape of the expanding arcade differs. The sheared arcade looks more rounded.

The shear velocity is a function of the latitude, so that field lines with foot points at different latitudes will be sheared at different velocities. This results in a non uniform stressing of the field lines. The imbalance between the magnetic tension and the magnetic pressure forces causes the flanks of the inner field lines to be pinched together. On the other hand, in the case of flux emergence, no additional azimuthal field is introduced in the system. The magnetic pressure/tension balance will involve just the poloidal component of the field, resulting in a more squared shape of the expanding arcade.

Figures 6 and 7 show the evolution of the azimuthal component of the current density, which is used as an indicator of the reconnection processes in the system. As consequence of the applied boundary conditions, the central arcade starts rising in both cases. As a result, the $X$-point is flattened and numerical reconnection sets in (Figs. 6a and 7a). This reconnection at the top of the expanding central arcade, known in the literature as breakout reconnection, removes the overlying field towards the side arcades. This will facilitate the upward motion of the central arcade. At a certain point in time, $t \simeq 19 \mathrm{~h} 50 \mathrm{~min}$ for the shearing case and $t \simeq 22 \mathrm{~h}$ for the flux emergence case, the single breakout reconnection site splits into two new sites at the flanks of the central arcade, causing the complete detatchment of the helmet streamer, resulting in the formation of a closed plasmoid, which will become the erupted structure. This process is described in detail by van der Holst et al. (2007). Figures $4 \mathrm{~b}$ and $5 \mathrm{~b}$ show the density configuration of the system after $t=20 \mathrm{~h} 54 \mathrm{~min}$ for the shearing case and after $t=24 \mathrm{~h} 35$ min for the flux emergence case. For both cases, this is the point at which the relative density within the detatched helmet streamer shows a significant increase.

For the shearing case, as the simulation is going on, the flanks of the erupting arcade are pushed closer together, allowing 


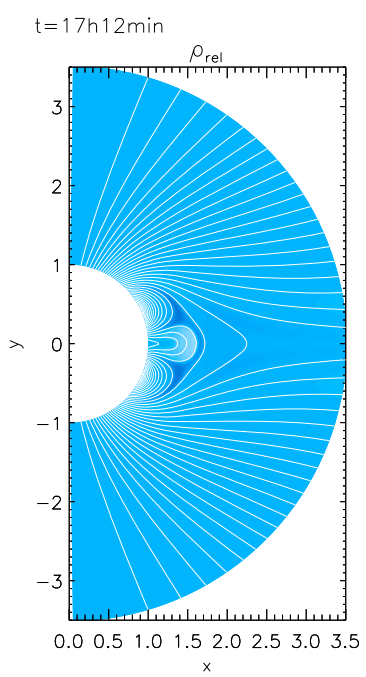

(a)

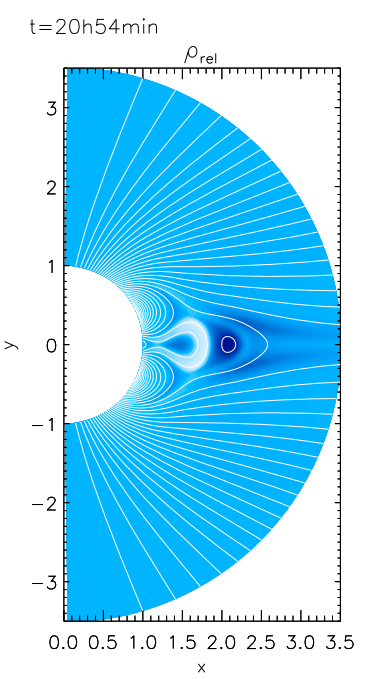

(b)

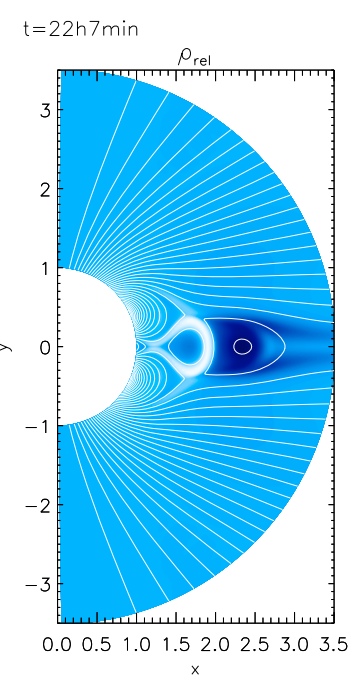

(c)

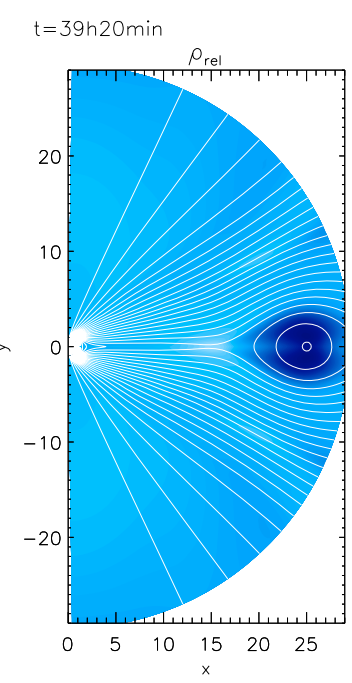

(d)

Fig. 4. Snapshots of the relative density and of the magnetic field lines for the foot point shearing case. Panel a) shows the expanding central arcade (17h12min). Panel b) shows the increase of the density inside the helmet streamer and the further expansion of the central arcade (20h54min). Panel c) shows the presence of the two flux ropes $(22 \mathrm{~h} 7 \mathrm{~min})$, and panel d) shows the actual CME leaving the computational domain (39h20min).

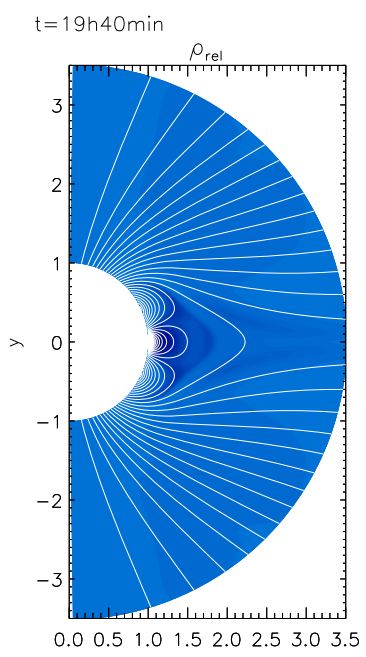

(a)

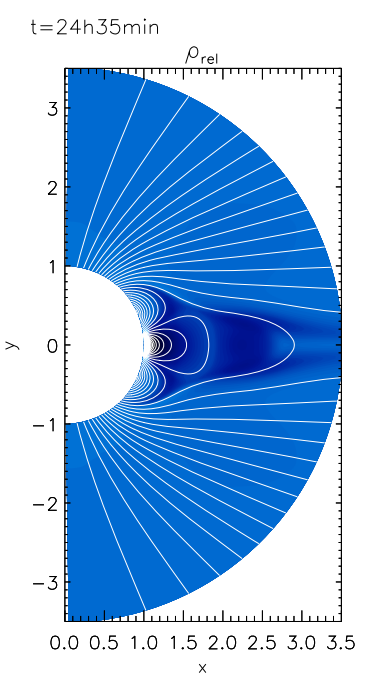

(b)

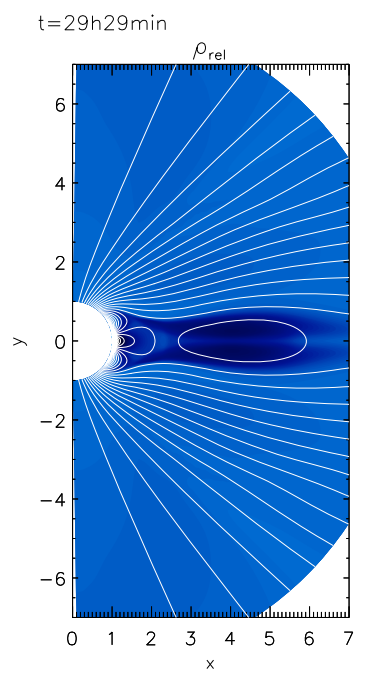

(c)
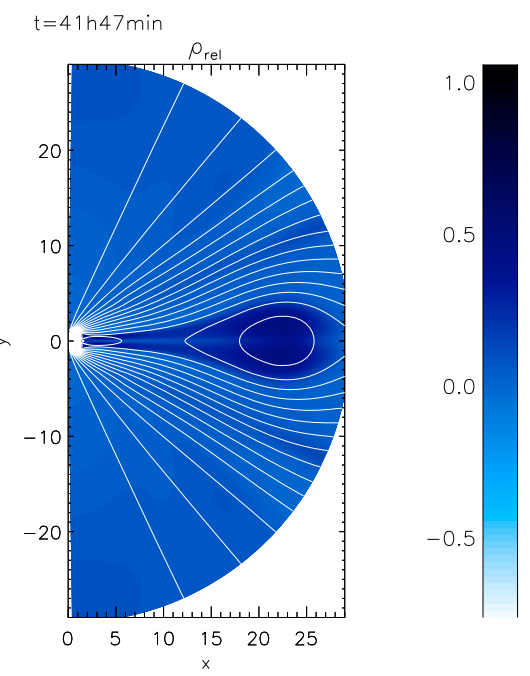

(d)

Fig. 5. Snapshots of the relative density and of the magnetic field lines for the flux emergence case. Panel a) shows the expanding central arcade (19h40min). Panel b) shows the increase of the density inside the helmet streamer and the further expansion of the central arcade when all the flux has emerged $(24 \mathrm{~h} 35 \mathrm{~min})$. The panels c) and d) show the actual CME propagating $(29 \mathrm{~h} 29 \mathrm{~min})$ and leaving the computational domain (41h47min)(Zuccarello et al. 2008).

reconnection to set in and eventually disconnecting the sheared arcade from the solar surface. This reconnection is visible in Figs. 6b,c and is referred to in the literature as flare reconnection. This dynamical process is the same as the breakout CME scenario. However, the inclusion of a background wind and helmet streamer makes the further evolution of the breakout CME different from the more idealised simulations of Antiochos et al. (1999); MacNeice et al. (2005). Figure 4c shows a more complex topology including two flux ropes. The leading flux rope, i.e. the detatched helmet streamer, has a magnetic field vector rotating in a clockwise direction. The magnetic field of the second flux rope, i.e. the detatched expanding central arcade, has a counter-clockwise orientation. At some point, the second flux rope will start reconnecting with the sides of the elongated helmet streamer. From now on, the flux of the breakout CME is partially transferred to the top of the helmet streamer and partly to the overlying field. The flare reconnection responsible for the formation of the second flux rope in the shearing case, is not observed in the flux emergence case. The central arcade, as more flux is added through the boundary, just continues to expand transferring flux into the helmet streamer. When the CMEs leave the computational domain, they look identical for both initiation mechanisms (see Figs. 4d and 5d). 


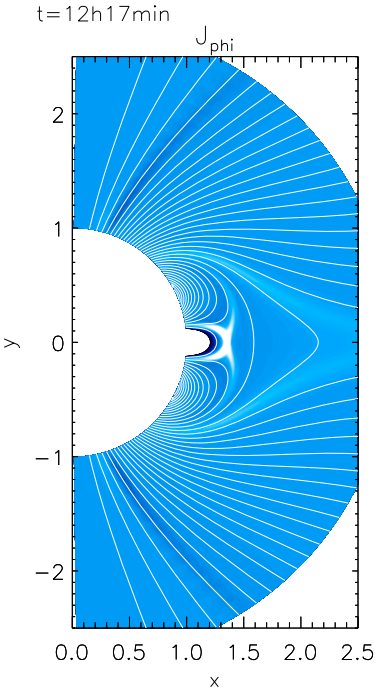

(a)

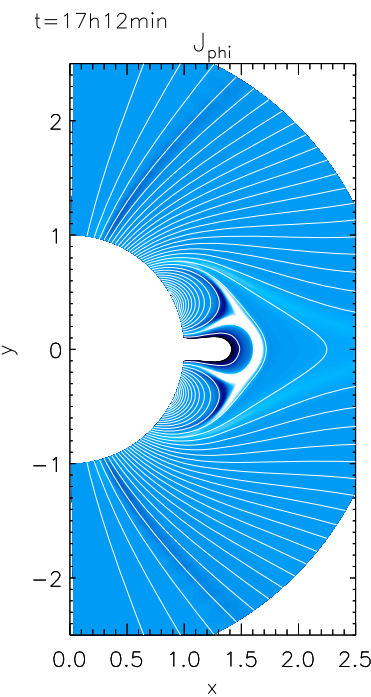

(b)

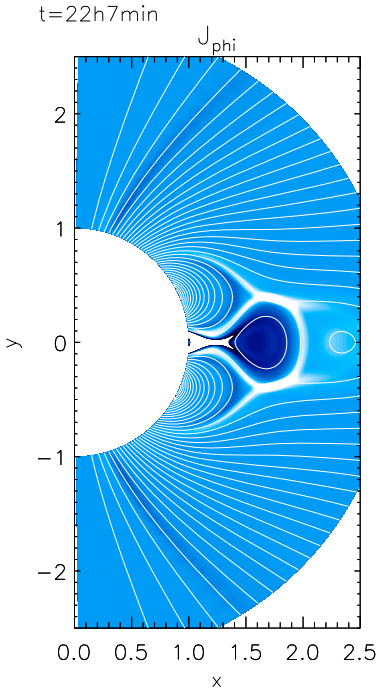

(c)

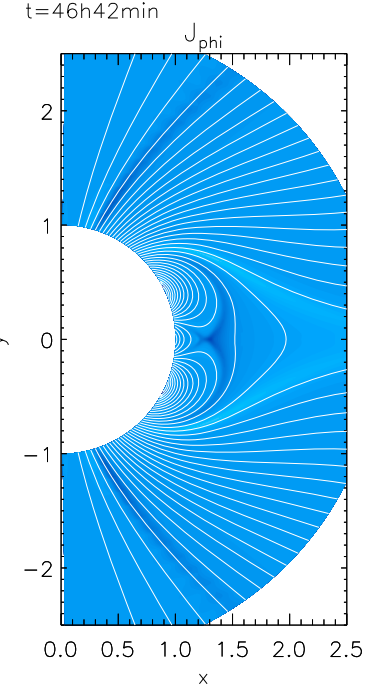

(d)

Fig. 6. Snapshots of the azimuthal component of the current density and of the magnetic field lines for the foot point shearing case. Panels a) and b) show the reconnection at the top of the expanding central arcade (12h17min and $17 \mathrm{~h} 12 \mathrm{~min}$, respectively) Panel c) shows the displacement of the reconnection site from the top of the central arcade to its flanks (22h7min). Panel d) shows how the system again reaches the initial equilibrium (46h42min).

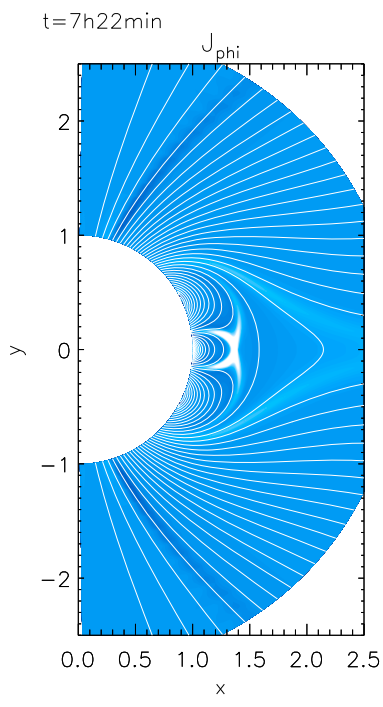

(a)

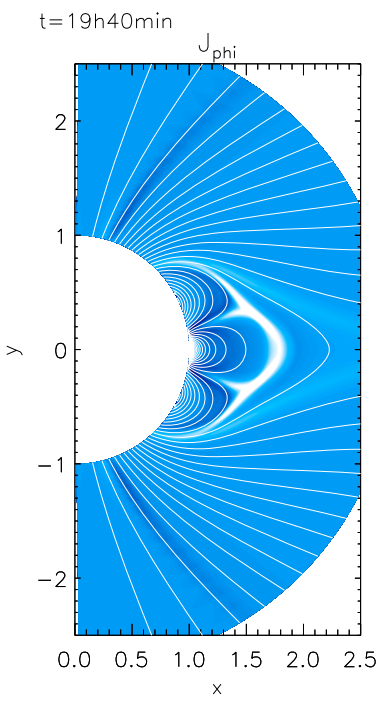

(b)

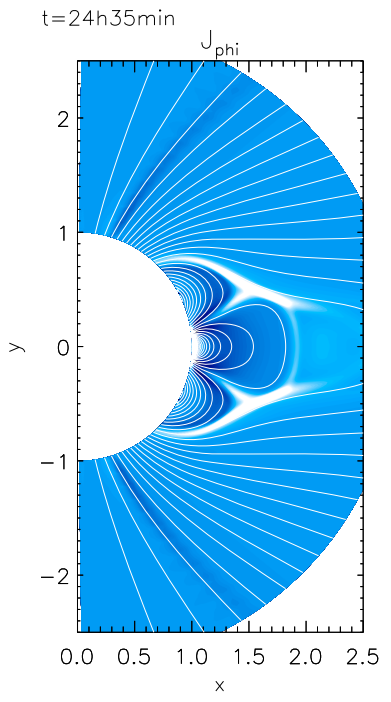

(c)

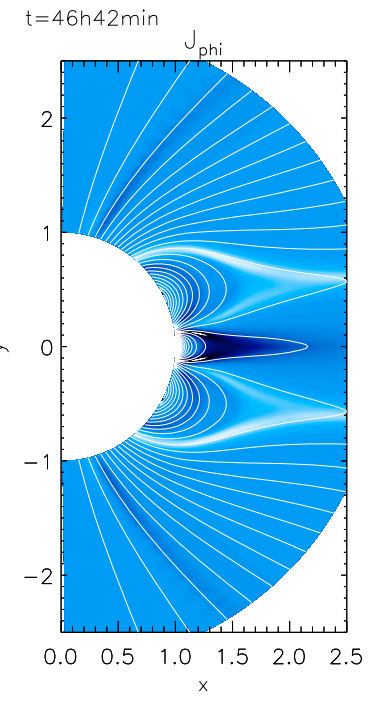

(d)

Fig. 7. Snapshots of the azimuthal component of the current density and of the magnetic field lines for the flux emergence case. Panels a) and b) show the reconnection at the top of the expanding central arcade ( $7 \mathrm{~h} 22 \mathrm{~min}$ and $19 \mathrm{~h} 40 \mathrm{~min}$, respectively). Panel c) shows the displacement of the reconnection site from the top of the central arcade to its flanks (24h35min). Panel d) shows how the system reaches a new equilibrium (46h42min) (Zuccarello et al. 2008).

The configuration of the system at the end of the simulations is shown in Figs. $6 \mathrm{~d}$ and $7 \mathrm{~d}$. After the time $\Delta t=24 \mathrm{~h}$ both shearing motions and flux emergence are stopped. In the shearing case, flare reconnection will enable the system to relax towards a more potential state. Because of the axial symmetry, this will result in minimising the azimuthal component of the field. Finally, the initial configuration is restored. For the flux emergence case, after the time interval $\Delta t$, the flux is kept constant at a different value than it was initially. No flare reconnection is observed so that the system can only relax to a new MHD equilibrium, in which the emerged additional flux in the central lobe raises its field volume so far out, eventually forming its own helmet streamer. The final configuration consists of three helmet streamers symmetrical around the equator.

\subsection{Dynamical evolution}

It has been generally accepted that the main energy source for CMEs is free magnetic energy. Regardless of the model or 

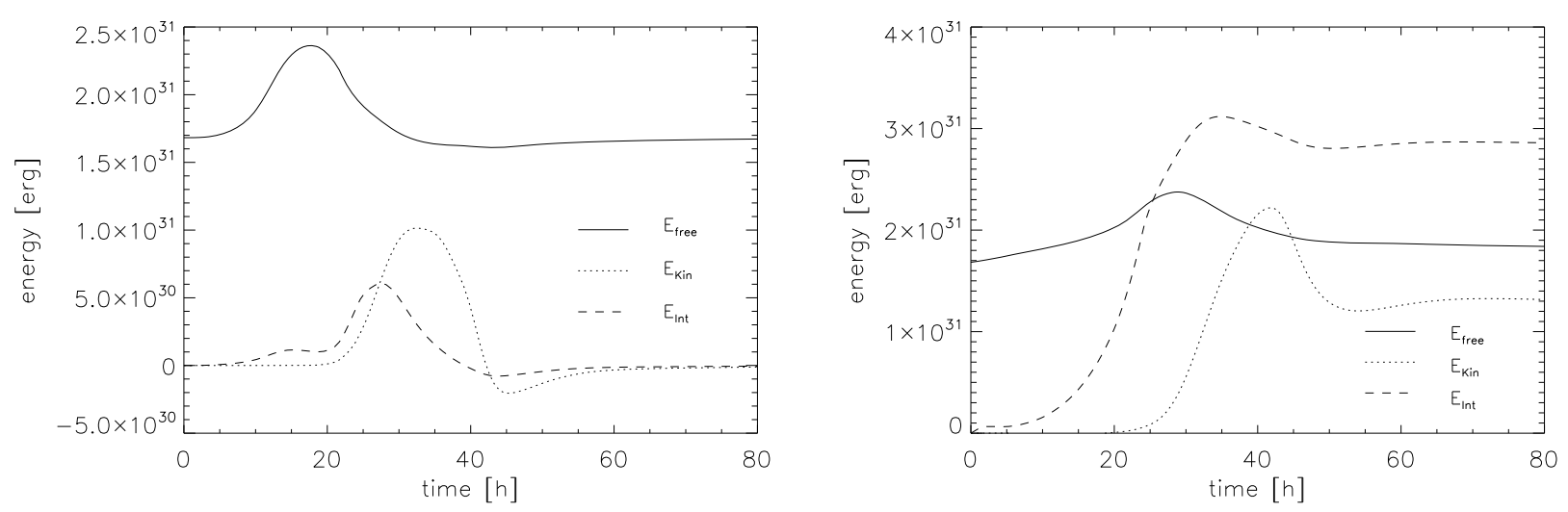

Fig. 8. Evolution in time of the free magnetic energy (solid line), the kinetic energy (dotted line), and the internal energy (dashed line) with respect to the energy of the background wind. Panel a): shearing case. Panel b): flux emergence case.

triggering mechanism used, one should therefore be able to convert free magnetic energy into the kinetic energy of the CME. The evolution of the kinetic energy and the internal energy with respect to the energy of the background wind, together with the evolution of the free magnetic energy, are shown in Fig. 8.

As can be deduced from Fig. 8, the initial configuration is non-potential, as in fact there is already a certain amount of free magnetic energy stored in the system. In both cases, as a consequence of the applied boundary conditions, an increase in the free magnetic energy is seen. Although we applied the boundary conditions over the same time interval, the maximum of free magnetic energy is obtained at $t=18 \mathrm{~h}$ for the shearing case and at $t=28 \mathrm{~h}$ for the flux emergence case. This difference occurs mainly because the maximum in the shear profile is reached at $t=12 \mathrm{~h}$, so that most of the energy storage occurs within the initial $2 / 3$ of the total shearing time. For flux emergence, the flux increases linearly with time over the time interval $\Delta t$. In both cases, when a decrease in free magnetic energy is seen, an increase in kinetic energy is observed. The maximum in kinetic energy corresponds to the time at which the CME starts to leave the computational domain. The time interval between the maximum in free magnetic energy and the maximum in kinetic energy is $12 \mathrm{~h}$ in both cases. This means that the time it takes for the CME to travel to the outer boundary of the computational domain is the same for both initiation mechanisms.

Figure 8 also shows the evolution of the internal energy for both cases. The evolution of this energy significantly differs depending on the driving mechanism applied. The maximum in the internal energy for the flux emergence case is six times higher than the shearing case. The emergence of new magnetic flux, more specifically the emergence of a radial field component, will lift material from the solar surface upward and fills up the inner arcade. This will increase the density in the central lobe with the consequent increase in the plasma pressure and in the internal energy. However, the relative position of the maxima in the internal energy with respect to the maxima in free magnetic energy and in kinetic energy is similar in both cases. After the free magnetic energy is efficiently released in the system, and before the CME leaves the numerical domain (maximum in kinetic energy), the conversion from internal energy into kinetic energy continues. The energy in the system at the end of the simulation is different for the two cases. After the shearing motions are stopped, the system will eventually return to its initial state and the energies return to their original values. In the flux emergence case, however, the system will relax to a new equilibrium having
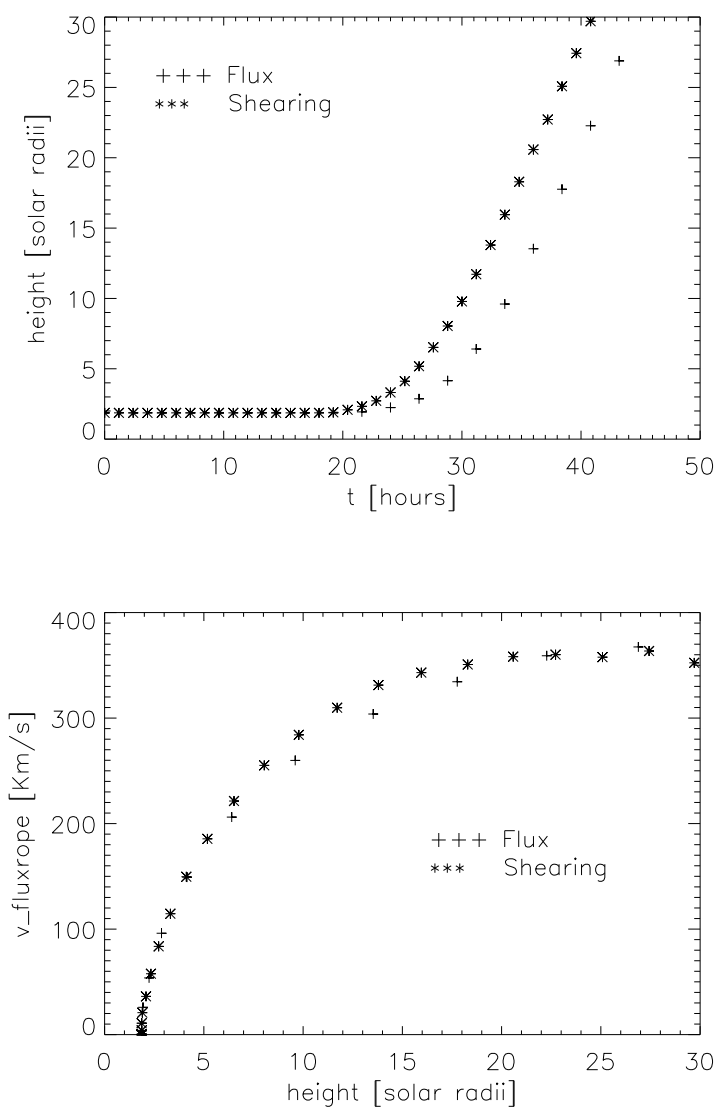

Fig. 9. Height-time (top panel) and velocity-height (bottom panel) plots of the ejected helmet streamer for both the shearing case (*-symbols) and the flux emergence case (+-symbols).

higher energy than the initial configuration due to the modified magnetic field at the boundary. The emerged poloidal flux remains rooted to the base of the corona.

Figure 9 shows the height-time plot (top panel) and the velocity-height plot (bottom panel) for both the shearing and the flux emergence case. The two curves are very similar. In the shearing case, the CME is launched, as already discussed, earlier than in the flux emergence case. However, the propagation of the CMEs through the numerical domain is identical from the 

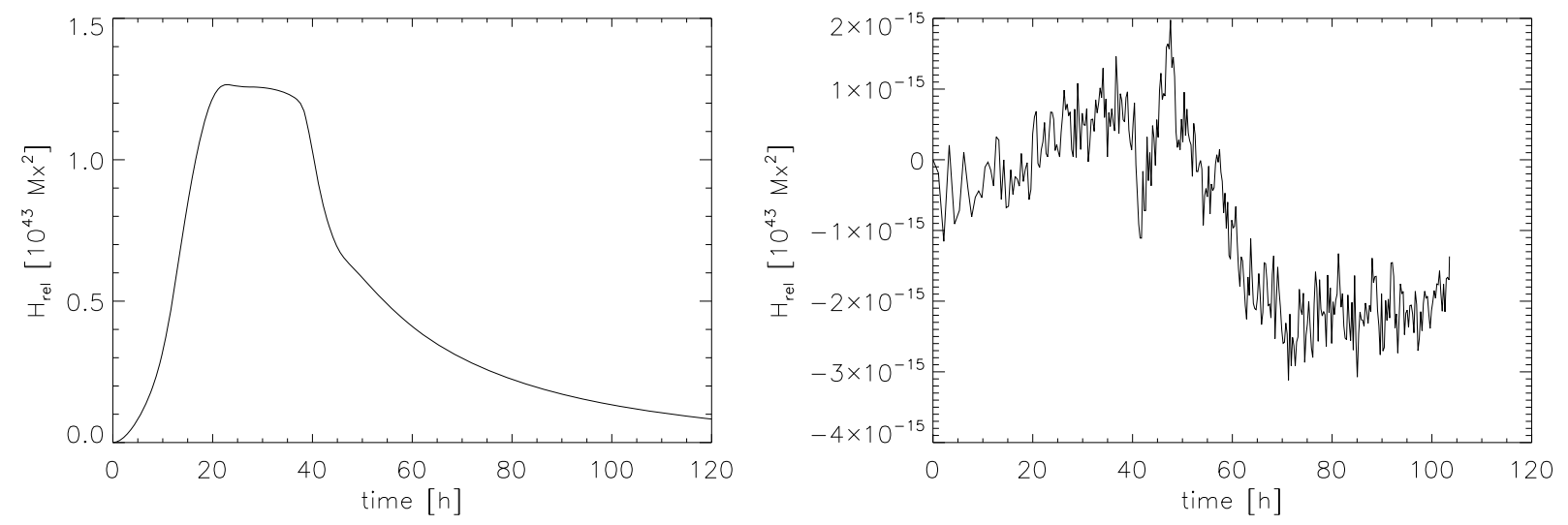

Fig. 10. Relative helicity evolution for the shearing case (panel $(a))$ and flux emergence case (panel $(b))$. For the flux emergence case, the fluctuations are purely numerical, so the helicity remains physically zero at all time.

kinematic point of view. These simulations show that the evolution of the actual CME is not dependent on which mechanism drives the eruption. In both simulations the terminal velocity of the CME is about $380 \mathrm{~km} \mathrm{~s}^{-1}$. This value agrees with the median speed of CMEs inferred from the observations (Zhang \& Low 2005). These simulations also suggest that neither the breakout model nor the flux emergence model could explain the minority of fast $\left(>1000 \mathrm{~km} \mathrm{~s}^{-1}\right) \mathrm{CMEs}$, at least in the parametric regimes explored.

\subsection{Relative magnetic helicity}

Berger \& Field (1984) showed that the magnetic helicity of the corona should be conserved during either an ideal or a resistive evolution of the coronal magnetic field. Consequently, photospheric motions will continuously inject helicity into the corona. The only way this helicity can be released is by erupting helical structures (Phillips et al. 2005). In their numerical simulations Kusano et al. (2004) find that eruptions are triggered when reversed magnetic helicity is injected into the system. Jacobs et al. (2006) used 2.5D ideal MHD simulations to investigate the effect of the background wind and the shear velocity on the initiation and evolution of a CME. They conclude that the amount of relative helicity present in the coronal volume plays an important role in the onset of the instability. Shiota et al. (2008) investigated the quantitative relationship between the magnetic flux of solar coronal arcades and the magnetic helicity injection, which is caused by shearing motions, in a spherical axisymmetrical domain. The authors find that the tearing mode produces plasmoids, whose elevation is almost proportional to the total amount of magnetic helicity contained in the arcade, and it is too slow to explain the CMEs' triggering process. They conclude that it may be difficult to trigger a CME just by the axisymmetric shearing motions and that some other mechanisms should be involved in the triggering process of a CME. Phillips et al. (2005) investigated the role of magnetic helicity in the framework of the breakout model. The authors compare the results of two simulations with slightly different shearing profiles, such that there is injection of magnetic helicity in one case while there is not in the other case. They demonstrate that, in their simulations, a key role is played by the free magnetic energy pumped in the system.
In this section, we discuss the role of relative helicity in our simulations. The relative magnetic helicity, expressed in a gauge invariant form, is defined as

$H_{\mathrm{rel}}=\int\left(\boldsymbol{A}+\boldsymbol{A}_{\mathrm{p}}\right) \cdot\left(\boldsymbol{B}-\boldsymbol{B}_{\mathrm{p}}\right) \mathrm{d} V$

where $\boldsymbol{B}$ is the magnetic field, $\boldsymbol{A}$ the magnetic vector potential, $\boldsymbol{B}_{\mathrm{p}}$ the potential magnetic field that, at the boundary, has the same distribution as $\boldsymbol{B}$, and finally, $\boldsymbol{A}_{\mathrm{p}}$ is its corresponding vector potential. Because of the chosen symmetry, according to Antiochos et al. (2002) the previous expression simplifies to

$H_{\text {rel }}=2 \int A_{\phi} B_{\phi} \mathrm{d} V$,

where $A_{\phi}$ and $B_{\phi}$ are the azimuthal components of the magnetic vector potential and of the magnetic field, respectively. This quantity is computed in all the simulations performed. Figure 10 shows the evolution of the relative helicity for both shearing and flux emergence case. As is evident in the latter case, the fluctuations in the helicity are purely numerical, so the helicity during the flux emergence experiment remains zero. On the other hand, the applied shearing motions inject magnetic helicity through the inner boundary during the time interval $\Delta t$. When the shearing motions are switched off and while the CME is propagating through the numerical domain, the helicity shows a plateau. Finally, when the eruption passes through the outer boundary, it carries with it a significant amount of magnetic helicity. These results show that (1) CMEs can carry away the magnetic helicity built up as a consequence of photospheric motions; (2) the injection of helicity is not a necessary constraint in the initiation of CMEs.

\subsection{Emerging dipole}

To further investigate the role of flux emergence and shearing motions in the initiation of CMEs, we performed one more simulation that combines the two mechanisms. Both the shearing motions and the emergence of new magnetic flux are still applied within the same region and time interval. By mixing the two initiation mechanisms, we intended to simulate, within the dimensionality limitations, the emergence of a new dipole within the existing active region, causing the original magnetic field to be stressed. During the emergence process, the shearing angle is dynamically changing. The effect of the combination of both 


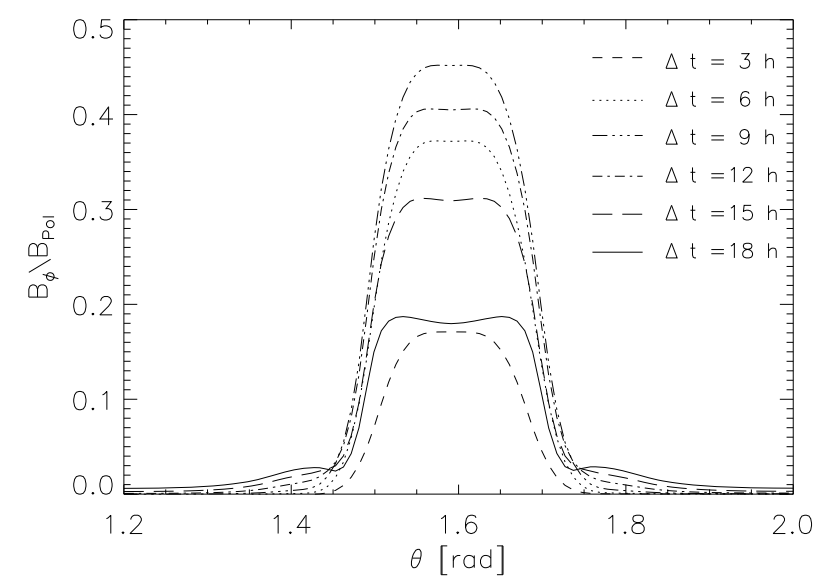

Fig. 11. Ratio of the azimuthal over the poloidal component of the field as function of colatitude and time.

mechanisms on the azimuthal component of the magnetic field at the inner boundary is plotted in Fig. 11. The ratio between the azimuthal and poloidal components of the magnetic field over the solar surface is plotted as a function of time. In the first half of the emergence time, the ratio is increasing almost uniformly within the central arcade. This ratio between the azimuthal and the poloidal component of the magnetic field is a measure of the obliqueness of the newly emerging field with respect to the original unsheared central arcade. The inclination of the emerging field varies with time, reaching a maximum angle of about $24^{\circ}$ after $10 \mathrm{~h}$. The decrease in the angle is then caused by both the increase in the poloidal component and by the reconnection process that essentially reduces the azimuthal component of the field.

For this particular simulation, a maximum shearing velocity of $v_{\max }=4.5 \mathrm{~km} \mathrm{~s}^{-1}$ was chosen and the total amount of emerged flux $\left(c_{\mathrm{e}}=-0.5\right)$ is $\Phi_{\mathrm{E}}=1.1 \times 10^{22} \mathrm{Mx}$. With those values for the shear speed and emergence rate, no CME is obtained when applying the initiation mechanisms separately. On the other hand, their combination efficiently energises the magnetic field enabling the build-up of both magnetic energy and helicity, eventually leading to an eruption.

Figure 12 shows the evolution of the system when both mechanisms are at work. When the flux emergence and the shearing motions are applied, the central arcade starts to expand. Throughout this phase, the morphology of the central expanding arcade is different from both the shearing and flux emergence cases. It is squared at the top, similar to the flux emergence case, but also introduces a significant pinching at the flanks. This results in a narrower and elongated shape of the central arcade. As in the other simulations presented here, the expansion of the central arcade leads to the detatchment of the helmet streamer resulting in the CME. After about $30 \mathrm{~h}$, the central arcade, as a consequence of the pinching of the field lines due to the shearing motions, detatches as well (see Fig. 12b). The height-time plot for this simulation shows the same general behaviour as that of the single cases, and the terminal velocity of the CME is still about $380 \mathrm{~km} \mathrm{~s}^{-1}$. The evolution of the reconnection sites is very similar to the one discussed for the shearing case, so we will not repeat that discussion here. Figure 13 shows the evolution of the energy with respect to the wind value. It is evident that the general dynamics are similar: the stress applied at the boundary

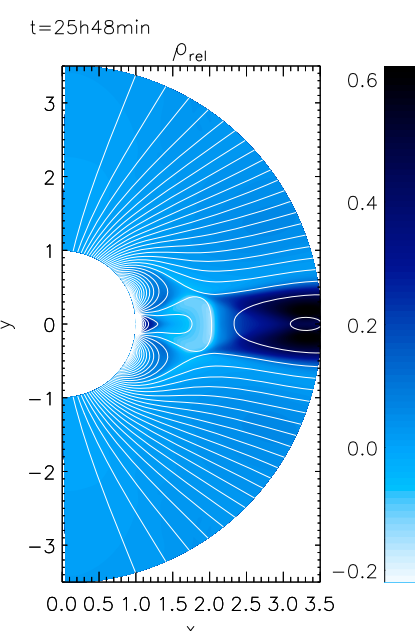

(a)

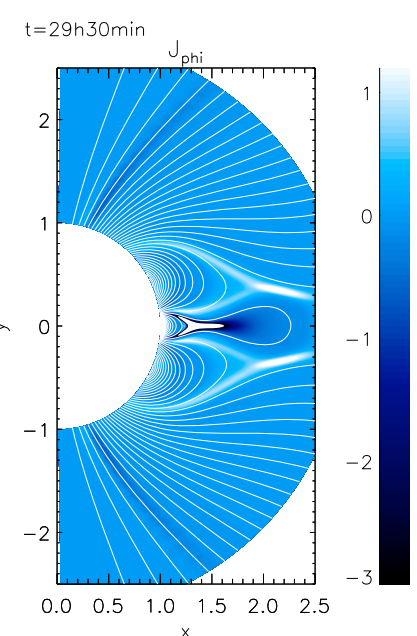

(b)
Fig. 12. Snapshots of the system. a) relative density (colour scale) and magnetic field lines (white). b) azimuthal component of the current density showing the location of the flare reconnection.

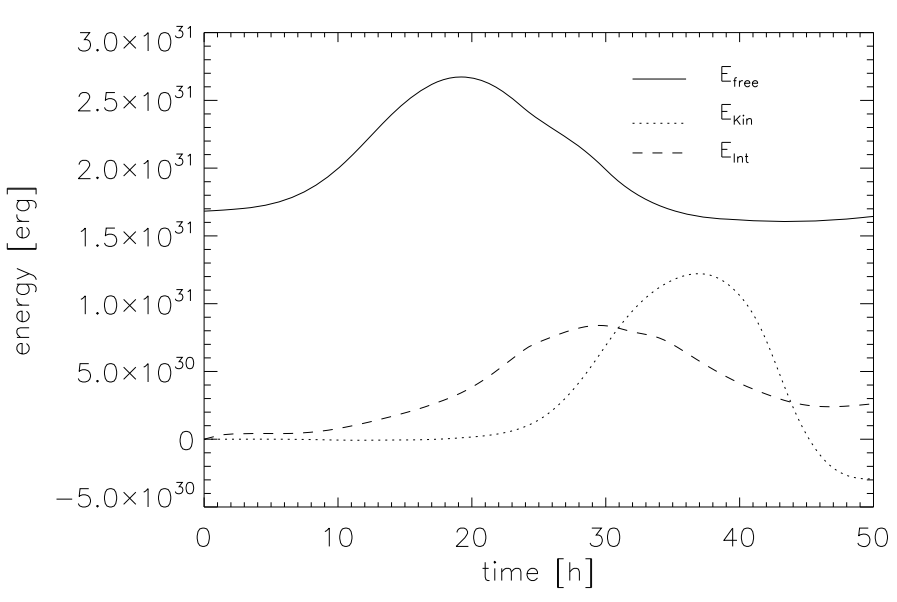

Fig. 13. Energy evolutions with respect to the wind values for both shearing and flux emergence. Solid line is the free magnetic energy, dotted line is the kinetic energy, and dashed line is the internal energy.

results in the increase in the free magnetic energy, which is then released and converted into kinetic energy. When the CME leaves the computational domain, the system relaxes to a new equilibrium having a slightly higher energy than the original system.

\section{Discussion}

CMEs are essentially magnetic phenomena. To understand their nature and to forecast CMEs, it is important to investigate the characteristics of the solar magnetic field prior to the CME occurrence. In Sect. 3.3 we already demonstrated that the injection of magnetic helicity into the system is not necessary for obtaining an eruption. However, from observations it is known that CMEs are often preceded by an evolution in the magnetic field during which the helicity budget of the source region increases.

To further investigate the role of magnetic helicity in the onset of CMEs, we performed several simulations like the one 


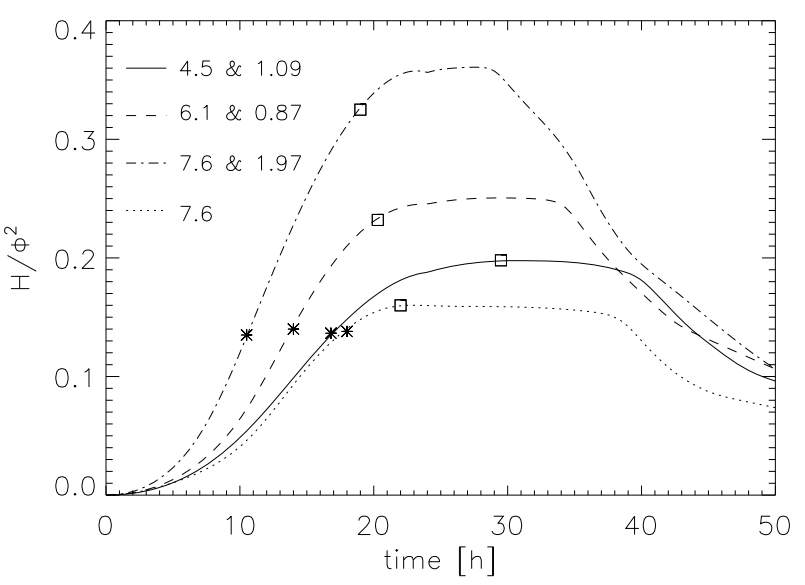

Fig. 14. Evolution of the relative helicity, expressed in non-dimensional units, over time. The results for different simulations are shown. Only shearing: $v_{\max }=7.6 \mathrm{~km} \mathrm{~s}^{-1}$ (dotted line); shearing and flux emergence: $v_{\max }=7.6 \mathrm{~km} \mathrm{~s}^{-1}$ and $\Phi_{\mathrm{E}}=1.97 \times 10^{22} \mathrm{Mx}$ (dash-dot), $v_{\max }=$ $6.1 \mathrm{~km} \mathrm{~s}^{-1}$ and $\Phi_{\mathrm{E}}=8.7 \times 10^{21} \mathrm{Mx}$ (dashes), and $v_{\max }=4.5 \mathrm{~km} \mathrm{~s}^{-1}$ and $\Phi_{\mathrm{E}}=1.09 \times 10^{22} \mathrm{Mx}$ (solid line). The $*$-signs represent the times of the helmet streamer detatchment, while the $\square$-signs indicate the moments when flare reconnection sets in.

presented in Sect. 3.4: helicity is injected into the coronal volume due to shearing motions between the opposite polarity foot points of a new emerging dipole. Apart from the simulation already presented in Sect. 3.4, two similar simulations were accomplished, namely combining the two simulations discussed in Sect. $3\left(v_{\max }=7.6 \mathrm{~km} \mathrm{~s}^{-1}\right.$ and $\left.\Phi_{\mathrm{E}}=1.97 \times 10^{22} \mathrm{Mx}\right)$ and one where we took the maximum shearing amplitude equal to $6.1 \mathrm{~km} \mathrm{~s}^{-1}$, and in total $8.8 \times 10^{21} \mathrm{Mx}$ of flux emerged. The time interval and the region over which the time dependent boundary conditions were applied is still the same as in the simulations discussed earlier, namely $\Delta t=24 \mathrm{~h}$ and $\Delta \theta=0.15$ radians.

When computing the amount of relative helicity in the simulation volume at the moment the helmet streamer detatches, it turns out that it is different for all the simulations. This is not surprising since the relative helicity is dependent on the magnetic field strength and this is different for each simulation, due to the difference in the flux emergence rate. However, when expressing the relative helicity in the more natural units of $H / \Phi^{2}$, where $\Phi$ is the total absolute magnetic flux, a threshold in the helicity seems to exist.

Figure 14 shows the change over time of the relative helicity in the coronal volume, expressed in the non-dimensional units of $H / \Phi^{2}$. Expressing the helicity in natural units leads to a direct measure of the stress of the field (Démoulin \& Pariat 2009), while observations show that $H \propto \Phi^{1.9 \pm 0.1}$ (Jeong \& Chae 2007; LaBonte et al. 2007). The figure also shows the evolution of the helicity for the simulation discussed in Sect. 3, in which only shearing motions are applied. The detatchment of the helmet streamer is indicated in the figure by the $*$-signs. The threshold value in relative helicity for the formation of the CME seems to be around $H / \Phi^{2}=0.14$. This value is in good agreement with observations (Démoulin \& Pariat (2009) and references therein) and previous simulations of shearing motion along the polarity inversion line for both young and old active regions (Démoulin et al. 2002). In the simulations of an emerging flux rope, Fan \& Gibson (2007) find that independently of the instability the system undergoes, the normalised relative helicity, at the moment of eruption, is between $0.16-0.18$. The authors

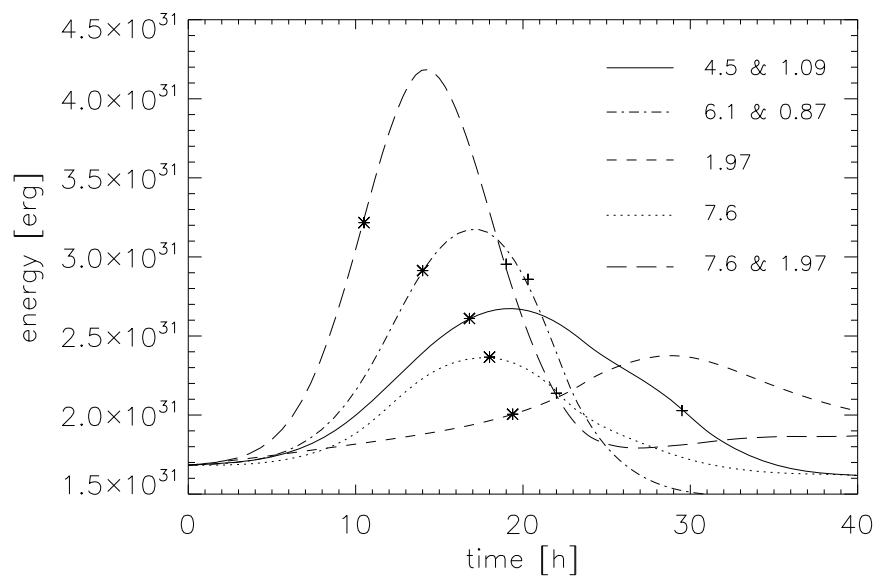

Fig. 15. Time evolution of the free magnetic energy as function of time. The different curves represent different simulations. Only shearing: $v_{\max }=7.6 \mathrm{~km} \mathrm{~s}^{-1}$ (dotted line); only flux emergence: $\Phi_{\mathrm{E}}=1.97 \times$ $10^{22} \mathrm{Mx}$ (dashed line); shearing and flux emergence: $v_{\max }=7.6 \mathrm{~km} \mathrm{~s}^{-1}$ and $\Phi_{\mathrm{E}}=1.97 \times 10^{22} \mathrm{Mx}$ (long dashes), $v_{\max }=6.1 \mathrm{~km} \mathrm{~s}^{-1}$ and $\Phi_{\mathrm{E}}=8.7 \times 10^{21} \mathrm{Mx}$ (dash-dotted line), and $v_{\max }=4.5 \mathrm{~km} \mathrm{~s}^{-1}$ and $\Phi_{\mathrm{E}}=1.09 \times 10^{22} \mathrm{Mx}$ (solid line). The $*$-signs represent the times of the helmet streamer detatchment, while the +-signs indicate the moments in which flare reconnection sets in.

interpret this as the result of a possible upper boundary in the total relative helicity as suggested by Zhang et al. (2006). In this context our results seem to confirm this conjecture further.

The $\square$-signs in Fig. 14 mark the moment at which the flare reconnection occurs. No threshold in helicity seems to exist for the latter. When applying purely shearing motions with a maximum amplitude of $4.5 \mathrm{~km} \mathrm{~s}^{-1}$, the eruption fails. The central arcade rises, but the overlying helmet streamer does not detatch. Investigating the helicity budget, it turns out that the amount of helicity in the coronal volume always stays well below the threshold value. On the other hand, it cannot be stated that the helicity threshold is the condicio sine qua non for obtaining a $\mathrm{CME}$, since the helicity is zero at all times for the flux emergence case of Sect. 3 and we do observe an eruption.

Both helicity and free magnetic energy are relevant quantities during the evolution of the system. In fact, the former is a measure of the stress of magnetic field due to plasma motions, while the latter not only takes this into account, but is also the amount of energy that can eventually drive the eruption.

Figure 15 shows the temporal evolution of the free magnetic energy for all the simulations discussed. The $*$-signs indicate the time at which the helmet streamer detatches, while the +-signs mark the time of the flare reconnection, if present. The amount of free magnetic energy built up in the system is different for every simulation and no clear threshold seems to exist. The differences in free magnetic energy could be related to the different morphology of the reconnection layers and to the different values of the Alfvén speed, which will influence the amount of energy stored before the breakout reconnection effectively sets in. However, in the cases where no CME was obtained (i.e. shearing amplitude of $4.5 \mathrm{~km} \mathrm{~s}^{-1}$ or flux emergence of $\left.1.1 \times 10^{22} \mathrm{Mx}\right)$, the amount of free magnetic energy was never more than $1.8 \times 10^{31} \mathrm{erg}$. This indicates that a minimum of energy is needed to be able to drive an eruption.

The duality between free magnetic energy and relative magnetic helicity accumulated in the system prior to the eruption has been investigated by Zhang et al. (2006) using a series of 
power-law axisymmetric force-free fields. The authors demonstrate that, in a closed domain, the magnetic helicity is a lower boundary for the free magnetic energy. They also argue that this result could be extended to the open corona. Their studies suggest a limiting value for the magnetic helicty beyond which a CME expulsion becomes unavoidable. Our simulations confirm this hypothesis for a more general magnetic field. In the simulations presented in this paper, at the moment of the eruption the system has enough free magnetic energy to drive the CME, although a threshold in this quantity is not clearly observed. The question is whether overcoming the magnetic helicity threshold without enough free magnetic energy would lead to a CME (Zhang et al. 2006). This is still an open question.

The helmet streamer detatchment is essentially a consequence of both the chosen magnetic topology and the expansion of the central arcade. All simulations presented in this work show the same evolution: the breakout reconnection transfers flux from the overlying field towards the side arcades leading to the detatchment of the helmet streamer. Up to this point, the visual differences between energising the corona by shearing of the magnetic foot points or by flux emergence are only related to the shape of the magnetic structures. The main difference between the two cases is the amount of helicity injected into the corona. The evolution of the two cases becomes different after the helmet streamer is ejected. In the shearing case, flare reconnection is observed after the CME occurred, while this is not present in the flux emergence case. These results demonstrate that, already within the same model, CMEs may occur without flares as observations suggest. In these simulations the flare reconnection plays a secondary role: the nature of the CME is the detatched helmet streamer, and it is not influenced by the flare reconnection. The helmet streamer is evacuated by the solar wind and becomes a slow CME, typically in observations referred to as a blowout CME (Howard et al. 1985).

We are confident that including the background solar wind together with the dimensionality limitation is the reason we do not obtain a real breakout eruption. In fact, when the arcade starts to expand, the breakout reconnection will detatch the helmet streamer before the flare reconnection sets in. This will result in the failure of the breakout process. Recently, van der Holst et al. (2009) have investigated the relation between blowout and breakout CMEs. The authors find that in a three-dimensional corona, if the central expanding arcade rises fast enough, the helmet streamer can swell up without detatching. The CME obtained in this case has a maximum velocity of about $620 \mathrm{~km} \mathrm{~s}^{-1}$ and can be classified as breakout. The main acceleration phase for this simulation is related to the flare reconnection. However, when the central arcade does not expand fast enough, the breakout reconnection detatches the helmet streamer, resulting in a slow blowout CME.

Robbrecht et al. (2009) report on a front side, large-scale CME of the blowout type without obvious counterparts in the corona. This CME could be only detected thanks to the two different viewpoints of the STEREO spacecraft. Their findings suggest that streamer blowouts may be hard to observe faceon and can be a possible explanation for "mystery" magnetic storms (i.e. storms without a clear solar origin). The CME event originated along a neutral line over the quiet Sun, and no large filament or active region was present prior to this event. The authors attribute the absence of any clear coronal signatures to the unusual large height at which the CME lifted off (the overlying loop system exceeds $1.4 R_{\odot}$ ). The observations of Robbrecht et al. (2009) are consistent with our simulations and point to the importance of blowout CMEs in space weather research.
Sheeley et al. (2007) have investigated the evolution of the helmet streamer detatchment during the 2006 July 1-2 event. Composite EIT/C2 (LASCO) running difference images show the swelling of the helmet streamer that eventually detatches. The images also show the formation of a $\mathrm{V}$-shaped density lack below the ejected material, indicative of a reconnection layer. Moreover, during these observations, no new flux emergence was observed. The authors propose a possible explanation for the formation of this current layer. When the expanding loops are carried away from the wind, their legs are stretched, pushed together and eventually reconnect. The observations of Sheeley et al. (2007) are consistent with our simulations when applying shearing motions.

Sheeley \& Wang (2007) studied about 160 streamer detatchment events. In particular, the authors investigated those CMEs that are related to the formation of what they call "in/out pairs". In the running difference images, the in/out pairs appear like an inward and outward moving feature having leading-white and trailing-black signatures, indicating plasma compression during, for example, a loop expansion. This expansion will result in the slow detatchment of the helmet streamer that will cause a blowout CME. The authors argue that these pair formations indicate reconnection occurring higher in the solar corona during for example flux emergence events. No $\mathrm{V}$-shaped density lack is observed for these events. This behaviour is qualitatively similar to that of our system when new flux emerges in the solar corona. The authors conclude that in/out pairs and streamers blowout CMEs seem to be part of the same broad class of streamer eruption events. We share their point of view. In fact, as our simulations suggest, depending on which is the major source of energy, shearing motions or emergence of new magnetic flux, the same magnetic configuration can undergo a different evolution.

\section{Conclusions}

In this paper we have presented the results of numerical simulations, in which a reconstructed solar corona with a solar wind is made unstable by two different driving mechanisms, viz. emergence of new magnetic flux and shearing of the magnetic foot points. A multiflux topology was reconstructed within the helmet streamer, suitable for the breakout model to work. Both the shearing motions and emergence of new magnetic flux cause the central arcade to expand and reconnection at the top to set in. This breakout reconnection eventually detatches the helmet streamer that is swept away along with the slow solar wind. In the meantime, an increase in the density is observed within the detatched helmet streamer. This helmet streamer detatchment is the actual slow CME we obtain independently of the driving mechanism used. The role of the background wind is crucial in our simulations. On one hand, without background wind the emergence of new magnetic flux would not lead to any eruption. On the other hand, we are confident that it plays a role in determining the failure of the breakout CME when shearing motions are also considered.

After the eruption of the helmet streamer, the evolution of the system is no longer similar for the two driver mechanisms. For the shearing case, flare reconnection at legs of the central expanding arcade will result in the formation of another flux rope that will eventually merge with the detatched helmet streamer. On the other hand, no flare reconnection is present for the flux emergence case and the central arcade will expand and eventually relax to a new equilibrium. In fact, the emergence of new poloidal magnetic field increases the separation between the flanks of the central arcade, not allowing flare reconnection 
and eventually bringing the $2.5 \mathrm{D}$ breakout model to rest. Also, a simulation in which the emergence of new magnetic flux is simultaneously subjected to shearing motions has been investigated. In this case the morphology of the central arcade was found to show some characteristics linkable to the flux emergence and some other related to the foot point shearing. From height-time plots and energy-time plots, we demonstrated that the general dynamical behaviour of the system is similar in all cases. This again agrees with the fact that the actual CME is the ejected helmet streamer and not the expanding central arcade. Finally, we showed that helicity is not needed to obtain a solar eruption. However, if helicity is present, a threshold seems to exist for the onset of the CME.

Acknowledgements. F.P.Z. would like to thank E. Priest for the interesting and useful comments. We also thank the anonymous referee whose comments helped us to improve the quality of this paper. These results were obtained in the framework of the projects GOA/2009-009 (K.U. Leuven), G.0304.07 (FWO-Vlaanderen), and C 90347 (ESA Prodex 9). Financial support by the European Commission through the SOLAIRE Network (MTRN-CT2006-035484) is gratefully acknowledged. The numerical results were obtained on the HPC cluster VIC of the K.U.Leuven.

\section{References}

Amari, T., Luciani, J. F., Aly, J. J., Mikić, Z., \& Linker, J. A. 2003, ApJ, 585, 1073

Amari, T., Aly, J. J., Mikic, Z., \& Linker, J. 2007, ApJ, 671, L189

Amari, T., Luciani, J. F., Mikić, Z., \& Linker, J. A. 2000, ApJ, 529, L49

Antiochos, S., DeVore, C., \& Klimchuk, J. 1999, ApJ, 510, 485

Antiochos, S., Karpen, J., \& DeVore, C. 2002, ApJ, 575, 578

Archontis, V., \& Török, T. 2008, A\&A, 492, L35

Archontis, V., Hood, A. W., \& Brady, C. 2007, A\&A, 466, 367

Berger, M. A., \& Field, G. B. 1984, J. Fluid. Mech., 147, 133

Chen, P., \& Shibata, K. 2000, ApJ, 545, 524

Démoulin, P., \& Pariat, E. 2009, Adv. Space Res., 43, 1013

Démoulin, P., Mandrini, C. H., Van Driel-Gesztelyi, L., Lopez Fuentes, M. C., \& Aulanier, G. 2002, Sol. Phys., 207, 87

DeVore, C. R., \& Antiochos, S. K. 2008, ApJ, 680, 740

Ding, J. Y., \& Hu, Y. Q. 2008, ApJ, 674, 554

Dubey, G., van der Holst, B., \& Poedts, S. 2006, A\&A, 27, 159
Falconer, D. A. 2001, J. Geophys. Res., 106, 25185

Fan, Y., \& Gibson, S. E. 2004, ApJ, 609, 1123

Fan, Y., \& Gibson, S. E. 2007, ApJ, 668, 1232

Forbes, T. G., Linker, J. A., Chen, J., et al. 2006, Space Sci. Rev., 123, 251

Green, L. M., Démoulin, P., Mandrini, C. H., \& Van Driel-Gesztelyi, L. 2003, Sol. Phys., 215, 307

Groth, C. P. T., De Zeeuw, D. L., Gombosi, T. I., \& Powell, K. G. 2000, J. Geophys. Res, 105, 25053

Howard, R. A., Sheeley, Jr., N. R., Michels, D. J., \& Koomen, M. J. 1985, J. Geophys. Res., 90, 8173

Jacobs, C., Poedts, S., \& van der Holst, B. 2006, A\&A, 450, 793

Jeong, H., \& Chae, J. 2007, ApJ, 671, 1022

Klimchuk, J. A. 2001, in Space Weather, ed. P. Song, H. J. Singer, \& G. L. Siscoe (AGU), Geophys. Monograph Ser., 125, 143

Kusano, K., Maeshiro, T., Yokoyama, T., \& Sakurai, T. 2004, ApJ, 610, 537

LaBonte, B. J., Georgoulis, M. K., \& Rust, D. M. 2007, ApJ, 671, 955

Liu, Y., \& Hayashi, K. 2006, ApJ, 640, 1135

Lynch, B. J., Antiochos, S. K., DeVore, C. R., Luhmann, J. G., \& Zurbuchen, T. H. 2008, ApJ, 683, 1192

MacNeice, P., Antiochos, S. K., Phillips, A., et al. 2005, ApJ, 614, 1028

Manchester, W., Gombosi, T., Roussev, I., et al. 2004, J. Geophys. Res., 109, A01102

Martin, S. F., Dezso, L., Antalova, A., Kucera, A., \& Harvey, K. L. 1982, Adv. Space. Res., 2, 39

Parker, E. N. 1958, ApJ, 128, 664

Phillips, A. D., MacNeice, P. J., \& Antiochos, S. K. 2005, ApJ, 624, L129

Robbrecht, E., Patsourakos, S., \& Vourlidas, A. 2009, ApJ, 701, 283

Roussev, I. I., \& Sokolov, I. V. 2006, in Solar Eruptions and Energetic Particles, ed. N. Gopalswamy, R. Mewaldt, \& J. Torsti (AGU), Geophys. Monograph Series, 165, 89

Roussev, I. I., Lugaz, N., \& Sokolov, I. V. 2007, ApJ, 668, L87

Sheeley, N. R., \& Wang, Y.-M. 2007, ApJ, 655, 1142

Sheeley, N. R., Warren, H. P., \& Wang, Y.-M. 2007, ApJ, 671, 926

Shiota, D., Kusano, K., Miyoshi, T., Nishikawa, N., \& Shibata, K. 2008, J. Geophys. Res. (Space Phys.), 113, 3

Sterling, A. C., Harra, L. K., \& Moore, R. L. 2007, ApJ, 669, 1359

Tóth, G. 1996, Astrophys. Lett. \& Comm., 34, 245

van Ballegooijen, A. A., \& Martens, P. C. H. 1989, ApJ, 343, 971

van der Holst, B., Jacobs, C., \& Poedts, S. 2007, ApJ, 671, L77

van der Holst, B., Manchester, W., Sokolov, I. V., et al. 2009, ApJ, 693, 1178

Yang, G., Xu, Y., Cao, W., et al. 2004, ApJ, 617, L151

Zhang, M., \& Low, B. C. 2005, A\&ARA, 43, 103

Zhang, M., Flyer, N., \& Low, B. C. 2006, ApJ, 644, 575

Zhang, Y., Zhang, M., \& Zhang, H. 2008, Sol. Phys., 250, 75

Zuccarello, F. P., Soenen, A., Poedts, S., Zuccarello, F., \& Jacobs, C. 2008, ApJ, 689, L157 